\title{
4D CHARACTERIZATION OF SHORT- AND LONG-TERM HEIGHT-VARYING DECORRELATED FOREST SAR BACKSCATTERING
}

\author{
Fabrizio Lombardini ${ }^{(1,2)}$, Federico Viviani ${ }^{(1,2)}$ \\ ${ }^{(1)}$ Dept. of Information Engineering, University of Pisa, via G. Caruso 16, 56122 Pisa (Italy) \\ ${ }^{(2)}$ RaSS Nat. Lab., CNIT, galleria G.B. Gerace 18, 56124 Pisa (Italy)
}

\begin{abstract}
Pol-InSAR and 3D multibaseline SAR Tomography (Tomo-SAR) can extract rich information on complex scenarios with multiple scatterers mapped in the SAR cell, in particular for forest remote sensing. However, forest scenarios are characterized by a temporal decorrelating volume canopy scatterer, and a set of related open problems exists, in particular for Tomo-SAR techniques to be applied to spaceborne monitoring of biomass. Multipass 4D Differential Tomography (Diff-Tomo) is a promising advancement, furnishing space (height)-time signatures of multiple scatterer dynamics in the SAR cell, originally with urban applications. In this paper, to better characterize forest decorrelation phenomena impacting Tomo-SAR/Pol-InSAR, experimental results are presented of the extension of Diff-Tomo methods for analyzing vegetated scenes, to extract jointly geometric and dynamic information of forest layers, at both the long and short time scale. The Diff-Tomo enabled functionality of separation in the height dimension of different temporal coherence levels ("coherence profiling") that are mixed (undiscriminated) in classical total coherence analyses is extensively applied to airborne P-band multipolarimetric data, and results of this investigation are shown. Also, first ground-based radar results are presented of an innovative profiling along the height dimension of the short-term coherence, in particular aiming to characterize the magnitudes of short-term coherence times. Their expected variability along the tree structures is confirmed for the first time.
\end{abstract}

Index Terms - SAR, tomography, volume scattering, vegetation, temporal decorrelation, multidimensional signals

\section{FOREST DECORRELATION AND TOMOGRAPHIC FRAMEWORKS}

In the last decade, advanced SAR methods based on acquisition diversity (in particular multipolarization or multibaseline interferometric data) and complex (amplitude and phase) value level processing have been developed, which are leading to next generation SAR products of $3 \mathrm{D}$ kind for imaging of multiple layover or volumetric scatterers [1-5]. Compared to classical SAR Interferometry, these techniques can produce more features for accurate and unambiguous geophysical parameter extraction.

In this context, multibaseline (MB) SAR Tomography (Tomo-SAR) is of increasing importance. Tomo-SAR coherently combines SAR data from a MB array typically synthesized by repeated passes, to obtain, through spatial (baseline) spectral estimation for elevation beamforming, profiles of the backscatterer intensity along the third dimension [1-5]. Beyond layover solution in garbled urban/infrastructure areas [2], and recent ice investigations [6], emerging tomographic remote sensing applications regard forest scenarios [3,4,7-9], e.g. tree height and biomass estimation, sub-canopy topographic mapping, and search-and-rescue/surveillance. However, these scenarios are characterized by temporal decorrelating (canopy) scatterers, and an open problem exists of possible height blurring and accuracy losses for TomoSAR techniques - and Polarimetric Interferometry (Pol-InSAR) - to be applied [7], in particular to biomass inventory and monitoring, relevant for the carbon cycle problems. Notably, the partial coherence can significantly impact not only spaceborne implementations of forest TomoSAR based on monostatic SAR systems, like in the ESA BIOMASS and NASA-ISRO NISAR projects, which can be affected by both long-term and short-term temporal decorrelation; also companion (or tandem) satellites systems, such as the investigated SAOCOM-CS, even adopting 3D correlative (track-pair only) imaging processing [8,9], can still be sensibly influenced by short-term decorrelation of the typical windblown vegetation, depending on the design of their formation-flying configuration time lag. In addition to other emerging non-stationarity issues related to seasonal and weather effects [4], it is thus important to extend current characterizations of forest temporal decorrelation, at both the long- and short-time scales, also accounting for the height-varying nature of the decorrelation phenomena $[7,10,11,12]$.

Recently, the more general unified framework of (4D i.e. 3D+Time) "Differential Tomography" (Diff-Tomo) [13] has been introduced ${ }^{1}$, which handles jointly the spatial (baseline) and the temporal dimensions synergically integrating the 3D Tomo-SAR with the Differential Interferometry concepts. This new coherent data combination framework allows, through proper spatialtemporal spectral estimation, the production of "space (height)-time" signatures of multiple scatterers inside the SAR cell. In particular, it enables joint resolution of multiple heights and (subsidence) deformation velocities of layover scatterers; this has got many applications to

\footnotetext{
${ }^{1}$ UniPi patent
} 
monitoring urban areas [2]. However, 4D Diff-Tomo also enables very advanced "Volumetric Differential Interferometry" capabilities, in which continuous profiling may be possible of velocity vs. height, as first hinted in [7]. More generally, Diff-Tomo can identify for the scattering components distributions of harmonics in the domain of spatial (height) and temporal frequency, the temporal harmonics constituting a Fourier decomposition of the signal from a scatterer with temporal decorrelation [7]. This also allows avoiding their misinterpretation in spatial-only 3D Tomo processing, a new concept this introduced in [7].

In this paper, latest developments carried out at UniPi and CNIT/RaSS are presented of advanced Diff-Tomobased analyses of decorrelating volumetric forest scenarios. The employed methods extract jointly geometric (structural) and dynamic information on the forest layers, thus dealing with the limitations of conventional ensemble coherence analyses. In fact, stratigraphic temporal decorrelation analyses are important, since TomoSAR/Pol-InSAR blurring mechanisms are local in height, an issue this considered also in the (limited coverage) TropiScat campaign [5].

In particular, the concept of space-time signatures of temporal decorrelation $[7,16]$-a recent new vision in SAR Interferometry- is exemplified with non-parametric DiffTomo analysis of BioSAR-1 ESA campaign P-band forest data, acquired by the DLR E-SAR airborne platform over a boreal region in Sweden. Then, a very large-scale P-band parametric Diff-Tomo [16] separation of height-varying long-term temporal decorrelation mechanisms is presented of the boreal area airborne data, with multiple polarizations.

Finally, concerning forest short-term temporal decorrelation $[7,11,14,16]$, in this work first results are presented of an innovative short-term decorrelation profiling along the height dimension, in particular aiming to characterize the magnitudes of short-term coherence times (needed to reach the short-term steady-state coherence levels), with emphasis on their expected variability along the tree structures $[7,11]$ and with the wind conditions. To this aim, extending the setup in [16], a first small scale experiment has been carried out using a fast sampling ground-based miniradar with electronic scanning, producing a unique "quick Diff-Tomo" characterization of short-term forest decorrelation processes.

\section{DIFF-TOMO VIEW OF TEMPORAL DECORRELATION}

According to the concept of space-time signature of a temporal decorrelating volume over ground in $[7,16]$, each scattering component subject to temporal decorrelation originates a band of temporal harmonics, in which the temporal perturbated signal can be decomposed, associated to the spatial frequency $f_{S}$ corresponding to the height of the component. This can be formulated through the signal theory Wiener-Khintchine theorem: the scattering component autocorrelation function in the time domain corresponds to a power spectral density (PSD, hereafter called spectrum for sake of simplicity) in the temporal frequency $\left(f_{T}\right)$ domain through a Fourier transform relation. It is worth noting here that these temporal spectra can be originated by both long-term and short-term decorrelation mechanisms, although physically (scatterer birth/death and growth motions, and wind action, respectively) and time-scale different.

Notably, the corresponding distributions of spatial (height) and temporal frequencies (the latter not merely coding subsidence velocitiy anymore) can be identified by Diff-Tomo, as far as the baseline-time sampling pattern and the adopted (superresolution) [13] spatial-temporal spectral estimator allow, as first exemplified by simulations in $[7,16]$. In particular, classical (airborne) monostatic acquisitions can be enough to get stratigraphies of long-term decorrelation; Diff-Tomo can also identify signatures of short-term decorrelation originating at different forest layer heights, in the multistatic or even better fully sampled acquisition case $[13,16]$. Thus forest temporal decorrelation, which is intrinsically a two-scale and height-varying random process, can be deeply and efficiently investigated through Diff-Tomo methods.

Recalling the real data verification of the space-time signature concept in [16], Fig. 1(a) reports a space-time signature (HH pol.), obtained by non-parametric superresolution 4D Diff-Tomo [13] in the height-temporal frequency domain $\left(P\left(f_{S}, f_{T}\right)\right.$ power distribution), from a multilook cell of the E-SAR P-band boreal BioSAR-1 data, 9 tracks, 2 month time span, about $20 \mathrm{~m}$ height Rayleigh resolution limit. Each cut along the temporal frequency is normalized to its peak, height by height, to highlight the different spectral spreads along the temporal frequency dimension. Markers of the scatterer heights (from the unnormalized power peak positions) have been superimposed. It is apparent the signature of long-term temporal decorrelating canopy with a bandwidth of temporal harmonics, originated by the temporal perturbations, wider than that of the ground, as expected from a quicker temporal decorrelation mechanism of the canopy. In Fig. 1(b), (normalized) temporal frequency spectra extracted from Fig. 1(a) associated to canopy and ground are shown: the different bandwidths of the two separated decorrelation mechanisms are apparent.

\section{EXTENSIVE SEPARATION OF P-BAND FOREST LONG-TERM TEMPORAL COHERENCE}

Regarding stratified analyses of forest long-term temporal decorrelation, large area characterizations can be notably obtained by advanced Diff-Tomo 4D or higher order (4D+) processing [13,14], starting from classical airborne multipass data with sparse baseline-time sampling. In fact Diff-Tomo has the potential to extract dynamic information in layered media, including separating in the height dimension different temporal decorrelation mechanisms that 
are mixed (undiscriminated) in the classical (ensemble) coherence analysis $[7,10,16]$. This separation is based on the concept of space-time signatures of long-term temporal decorrelation as mentioned before.

For an extended and quantitative discrimination and phenomenological analysis of the different temporal decorrelation levels of the canopy and ground scatterers, the automated semi-parametric superresolution Diff-Tomo method in [16] (AR temporal spectrum model) is applied here to a very large area of the P-band E-SAR data, with different polarizations ( $\mathrm{HV}$ and $\mathrm{HH}$ ). A Diff-Tomo fitting functional $P\left(f_{S}, \bar{f}_{T}, B_{f_{T}}\right)$, with $f_{S}$ and $\bar{f}_{T}$ the spatial frequency and the temporal frequency centroid, respectively, is exploited for temporal bandwidth estimation at each height, with $\bar{f}_{T}$ as a nuisance:

$$
\hat{B}_{f_{T}}\left(f_{S}\right)=\underset{B_{f_{T}}, \bar{f}_{T}}{\arg \max } P\left(f_{S}, \bar{f}_{T}, B_{f_{T}}\right) .
$$

This corresponds to a "5D" information extraction (rg., az., height, collective temporal phase shift rate, bandwidth).

The very large scale (500 land hectares) analysis of the different 5D-separated temporal decorrelation mechanisms is presented in Figs. 2 and 3, for $\mathrm{HV}$ and $\mathrm{HH}$ pol., respectively (for overall coherence down to 0.4 and, here, without LIDAR masking). Fig. 2(a) shows the estimated temporal bandwidth (phase cycles/month) for ground scatterers, and Fig. 2(b) that for canopy scatterers, at HV polarization. It is again apparent the typically higher temporal bandwidth (lower long-term coherence time) of the canopy layer. Corresponding average estimates of canopy and ground coherence times are 6 and 13.2 months, respectively, for $\mathrm{HV}$ pol. A few spotted zones with large bandwidths are affected by low overall coherence and SNR. In Fig. 3 the ground scatterer temporal bandwidth estimates are reported for $\mathrm{HH}$ pol. It is apparent how in this case the estimated bandwidths are typically lower than in Fig. 2(a); average separated ground coherence time resulted to be about three-fold rising in $\mathrm{HH}$ pol. with respect to $\mathrm{HV}$ pol., the phenomenological interpretation being consistent with the typical highly coherent co-pol scattering mechanism of the trunk-ground double bounce.

Noteworthy, the coverage of such 4D+ Diff-Tomo airborne analyses goes much beyond what achievable with tower-radar based TropiScat measurements [5] (4700 analyzed cells vs 160 possible cells from TropiScat which cover less than 1 hectare), and the Diff-Tomo processing method could be applied to incoming AfriSAR airborne data to extend AfriScat tower analyses.

\section{NOVEL SHORT-TERM TEMPORAL COHERENCE SEPARATION}

Concerning forest short-term temporal decorrelation $[7,11,14,16]$, in this work first results are presented of an innovative short-term coherence profiling along the height dimension, in particular aiming to characterize the magnitudes of short-term coherence times (needed to reach the short-term steady-state coherence level) with emphasis on their expected variability along the tree structures $[7,11]$. In fact, this is a currently unknown phenomenology, that can be of particular importance in the design of non-fully simultaneous companion satellite systems. Extending the setup in [16], a first small scale experiment has been carried out using a special ground-based miniradar (ack. Ids), currently at X-band, grazing angle, $2 \mathrm{~m}$ height Rayleigh resolution limit, $\mathrm{HH}$ pol., capable of extremely quick repeated acquisitions (adapted to produce hundreds of tomograms per second), that are exploited to produce a 4D "quick Diff-Tomo" characterization of short-term decorrelation processes.

The test scene is located in Pisa, Italy and comprehends a few poplar and elm trees, the wind condition being light breeze, cross-wind, and the acquisition season early autumn. The scenario is shown in Fig. 4. After data processing, first unique non-parametric space-time signatures of short-term temporal decorrelation have been derived for several heights of the tree as shown in Fig. 5. In particular, here, 3 temporal signatures for 3 heights are reported for a multilook cell of the data: bottom canopy, halfway canopy, and top canopy. It is apparent that shortterm temporal bandwidth is sensibly increasingly larger as the selected height in the canopy is higher, as it would have been expected from typical height-varying temporal decorrelation behavior and windblown tree/foliage motions. From these space-time short-term signatures, short-term coherence times for each canopy height $\left(\tau_{c}(h)\right)$ have been accurately measured by exponential temporal spectrum fitting, and they are shown in Fig. 6. Their values appear reasonable for the X-band (of the order of $50 \mathrm{msec}$ on the average) and short-term coherence time resulted to decrease by about $80 \%$ from the bottom of the canopy to the top.

To give a further demonstration of the capabilities of this new coherence characterization system, another spacetime signature of short-term temporal decorrelation from data acquired at a different time (1 hour later) is reported in Fig. 7, with the wind condition being now lighter breeze, still cross-wind. Other results are shown at the conference.

\section{CONCLUSIONS}

These largest analyzed area long- and first short-term phenomenological investigation methodologies and results can be employed to better characterize perturbation effects of forest temporal decorrelation on TomoSAR $[1,3,4,7]$ and Pol-InSAR [11,12], and to tune decorrelation-robust TomoSAR algorithms [14,16], or satellite system designs. In particular, they could be useful in the developments of the spaceborne forest remote sensing missions BIOMASS and SAOCOM-CS, related supporting measurements campaigns could be oriented accordingly, and the Diff-Tomo airborne and new quick Diff-Tomo miniradar investigations could be extended to L-/S-band, which is adopted in the NISAR mission project. 


\section{REFERENCES}

[1] A. Reigber, A. Moreira, "First Demonstration of Airborne SAR Tomography using Multibaseline L-band Data," IEEE TGARS, 38(5), pp.2142-2152, 2000.

[2] G. Fornaro, F. Serafino, F. Soldovieri, "Three Dimensional Focusing With Multipass SAR Data," IEEE TGARS, 41(3), pp. 507-517, 2003.

[3] M. Nannini, R. Scheiber, R. Horn, "Imaging of Targets Beneath Foliage with SAR Tomography," EUSAR'08.

[4] M. Pardini, K. Papathanassiou, "L-band Tomographic Monitoring of Temperate Forests with TangoSAT - Potentials, Challenges And Synergies," ESA Saocom Companion Satellite Workshop 2014.

[5] Dinh Ho Tong Minh, et al., "Vertical Structure of P-Band Temporal Decorrelation at the Paracou Forest: Results From TropiScat," IEEE GRSL, 11(8), pp.1438-1442, 2014.

[6] L. Ferro-Famil, S. Tebaldini, M. Davy, F. Boute, "3D SAR Imaging of the Snowpack at X- and Ku-Band: Results from the AlpSAR Campaign," EUSAR'14.

[7] F. Lombardini, F. Cai, "3D Tomographic and Differential Tomographic Response to Partially Coherent Scenes," IGARSS'08.

[8] R.N. Treuhaft, et al., "Tropical-Forest Density Profiles from Multibaseline Interferometric SAR," IGARSS'06.

[9] M. Pardini, K. Papathanassiou, "Robust Estimation of the Vertical Structure of Forest with Coherence Tomography," ESA PolInSAR '11 Workshop.

[10] F. Lombardini, F. Cai, "Evolutions of Diff-Tomo for Sensing Subcanopy Deformations and Height-varying Temporal Coherence," ESA Fringe'11 Workshop.

[11] M. Lavalle, S. Hensley, "Demonstration of Repeat-Pass POLINSAR Using UAVSAR: the RMOG Model," IGARSS' 12.

[12] M. Lavalle, M. Simard, S. Hensley, "A Temporal Decorrelation Model for Polarimetric Radar Interferometers," IEEE TGARS, 50 (7), pp.28802888, July 2012.

[13] F. Lombardini, "Differential Tomography: A New Framework for SAR Interferometry", IEEE TGARS, 43(1), pp.37-44, 2005.

[14] F. Lombardini, F. Cai, "Temporal Decorrelation-Robust SAR Tomography," IEEE TGARS, 52(9), pp.5412-5421, 2014.

[15] F. Lombardini, M. Pardini, "Superresolution Differential Tomography: Experiments on Identification of Multiple Scatterers in Spaceborne SAR Data," IEEE TGARS, 50(4), pp.1117-1129, 2012.

[16] F. Lombardini, F. Viviani, "Differential SAR Tomography Opportunities for Low-Frequency Companion Satellite Systems," ESA Saocom Companion Satellite Workshop 2014.

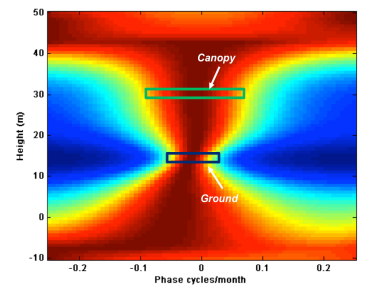

(a)

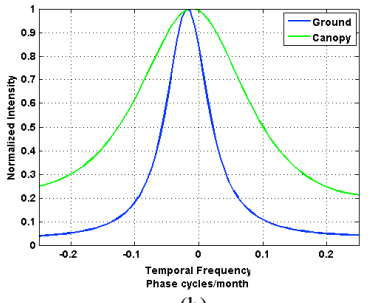

(b)
Fig. 1 - Long-term P-band decorrelation of a forested cell:

(a) normalized space-time signature;

(b) temporal harmonics distribution at ground and canopy heights

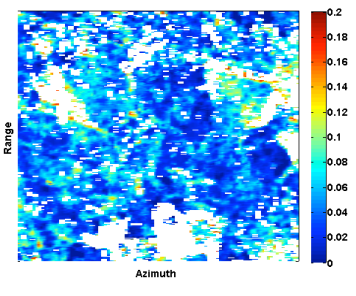

(a)

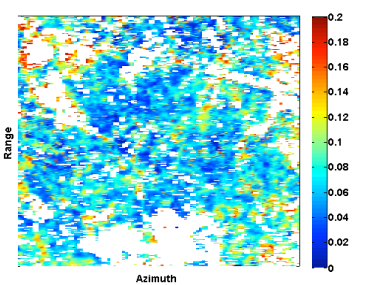

(b)
Fig. 2 - Separated temporal bandwidth maps (phase cycles/month), HV pol.: (a) ground height; (b) canopy height

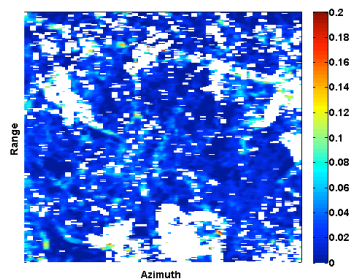

Fig. 3 - Separated temporal bandwidth map (phase cycles/month), $\mathrm{HH}$ pol., ground height

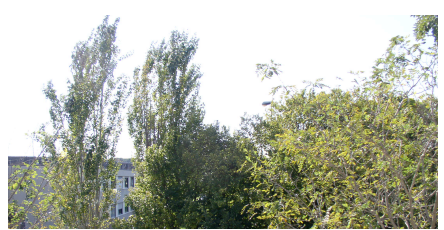

Fig. 4 - Tree scenario of the quick Diff-Tomo experiment carried out with the special ground-based X-band miniradar

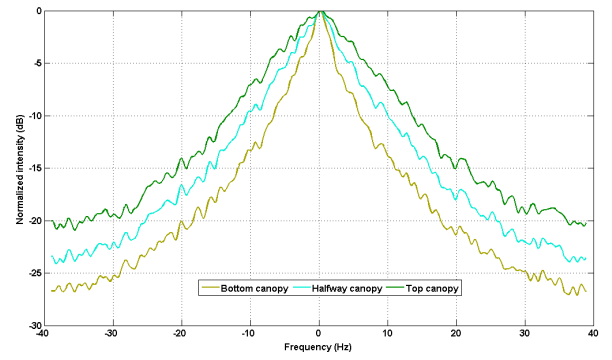

Fig.5 - Short-term height-varying decorrelation, tree cell, temporal harmonics distribution at the bottom, halfway, and top height of canopy

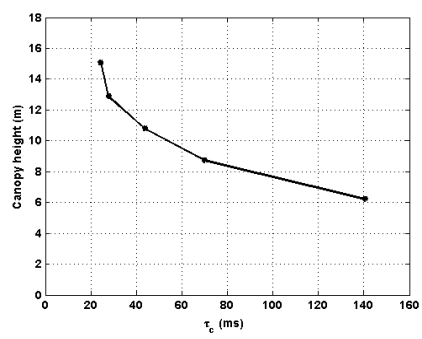

Fig.6 - Short-term height-varying coherence times for five different canopy heights, tree cell

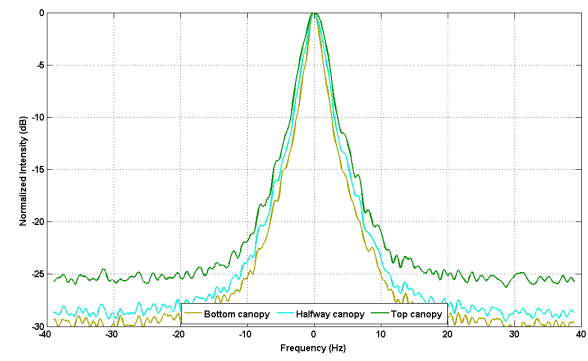

Fig.7 - Short-term height-varying decorrelation, tree cell (lighter wind), temporal harmonics distribution at the bottom, halfway, and top height of canopy 